\title{
Red Cell Antibody Screening: A Single Centre Experience
}

Naznin Muhammad ${ }^{1}$, Syifa Zainal Azhar ${ }^{1}$, Wan Husna Barakah ${ }^{1}$, Norlelawati A. Talib $^{1}$, Asmah Hanim Hamdan ${ }^{1}$, Azlina Abd. Fuaat ${ }^{1}$, Nor Zamzila Abdullah ${ }^{1}$, Shaik Farizan Shaik Daud ${ }^{1}$

${ }^{1}$ Department of Pathology and Laboratory Medicine, Kulliyyah of Medicine, International Islamic University Malaysia

Presenter: Naznin Muhammad

Introduction: The Transfusion Unit of International Islamic University Medical Centre (IIUM MC) performs screening for red cell antibodies for all patients who are to be or might be transfused with red cell components. There are two types of red cell antibodies, the alloantibodies and autoantibodies. While the red cell autoantibodies are produced against self-antigen, the alloantibodies are the results of sensitization through transfusion or pregnancies. These antibodies are implicated in haemolytic transfusion reactions. This retrospective study examines the prevalence of red cell antibodies among patients of IIUM MC. Materials and Methods: All group cross matching and group screen and hold testing performed in IIUM MC from January 2017 to June 2018 were reviewed. Results: In total, samples of 3,712 patients were subjected to antibody screening using gel card. Twelve patients $(0.32 \%)$ had positive antibody screening tests. The age ranged from 17 to 77 years. Nine (75\%) patients were females. Four patients had positivity with Screening Cell I, one patient with Screening Cell II and III respectively. In the remaining patients $(6 / 12)$ the antibody screening was positive for both Screening Cell I and II. In nine patients (0.24\%) alloantibodies were identified, with six exhibiting multiple antibodies. The alloantibodies identified include anti-Le(a), anti-Le(b), anti-Kp(a), anti-C, anti-c, anti-E and anti-e. In three patients autoantibodies with no specificities were detected. Conclusion: Although the prevalence of red cell antibodies, specifically the alloantibodies is lower compared to other studies, the finding further reiterates the need to screen for unexpected red cell antibodies prior to red cell transfusion. 